\title{
Effect of Inhomogeneous Heat Flow on the Enhancement of Heat Capacity in Helium-II by Counterflow near $\boldsymbol{T}_{\lambda}$
}

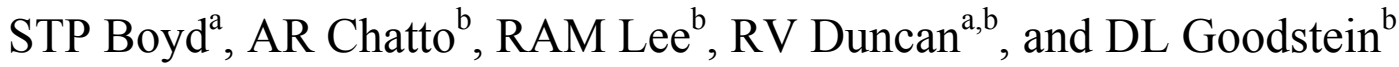 \\ ${ }^{a}$ Department of Physics and Astronomy, University of New Mexico, Albuquerque NM 87131, USA \\ ${ }^{b}$ Condensed Matter Physics, California Institute of Technology, Pasadena CA 91125, USA
}

\begin{abstract}
In 2000 Harter et al. reported the first measurements of the enhancement of the heat capacity $\Delta C_{Q} \equiv C(Q)-$ $C(Q=0)$ of helium-II transporting a heat flux density $Q$ near $T_{\lambda}$. Surprisingly, their measured $\Delta C_{Q}$ was $\sim 7-12$ times larger than predicted, depending on which theory was assumed. In this report we present a candidate explanation for this discrepancy: unintended heat flux inhomogeneity. Because $C(Q)$ should diverge at a critical heat flux density $Q_{c}$, homogeneous heat flow is required for an accurate measurement. We present results from numerical analysis of the heat flow in the Harter et al. cell indicating that substantial inhomogeneity occurred. We determine the effect of the inhomogeneity on $\Delta C_{Q}$ and find rough agreement with the observed disparity between prediction and measurement.
\end{abstract}

Keywords: helium, superfluid, heat capacity, lambda transition.

PACS: $67.40 . \mathrm{Kh}, 67.40 . \mathrm{Pm}, 64.60 . \mathrm{Ht}$

In order to evaluate the idea that unintended inhomogeneity of the heat flow in the Harter et al.[1] experiment might account for the discrepancy between measurement and predictions[2,3] of $\Delta C_{Q}$, we must estimate the heat flow field $\boldsymbol{Q}(\boldsymbol{r})$ in the helium-II. It is not difficult to show[4] that thermal counterflow in helium-II can be solved simultaneously with the diffusive heat flow in the enclosing experimental cell using a standard finite-element solver[5], if the helium-II is nondissipative, nonvortical, nearly isothermal, and free of net mass flow $(\boldsymbol{J}=0)$. These conditions should have been well-approximated in the Harter et al. experiment.

Such a numerical model has been constructed and solved for the Harter et al. cell. The model geometry is shown in Fig. 1. Not visible at this scale is the model for the Kapitza boundary resistance $R_{\mathrm{K}}$ : an artificial thin envelope of thickness $\delta=25 \mu \mathrm{m}$ and thermal conductivity $\kappa_{R K}=\delta / R_{\mathrm{K}}$ interposed between the helium and the cell walls.

For best accuracy, the helium-II diffusion coefficient should be modeled as $\kappa_{\mathrm{He}}=\alpha\left(\rho_{\mathrm{s}} / \rho_{\mathrm{n}}\right)$, where $\alpha$ is a large constant required to reduce the variation of the scalar superfluid velocity potential function[4]. However, Harter et al. had a very short cell $(0.64 \mathrm{~mm})$ and did not approach closer to $T_{\lambda}$ than $\sim 0.5 \times 10^{-6} \mathrm{~K}$, limiting the maximum variation of $\rho_{\mathrm{s}} / \rho_{\mathrm{n}}$ over the height of their cell to $\sim 10 \%$. To reduce the number of required computations we have approximated $\rho_{\mathrm{s}} / \rho_{\mathrm{n}}$ as constant and set $\kappa_{\mathrm{He}}=10^{6} \mathrm{~W} / \mathrm{cmK}$. Test reductions of $\kappa_{\mathrm{He}}$ to $10^{5} \mathrm{~W} / \mathrm{cmK}$ changed calculated enhancements by only $\sim 0.01 \%$, verifying that $\kappa_{\mathrm{He}}$ is sufficiently large.

To within their measurement noise, Harter et al. found that $t^{-\alpha} \Delta C_{Q}$ was linear in $\left(Q / Q_{c}\right)^{2}$, where $t=\left(T_{\lambda^{-}}\right.$ $T) / T_{\lambda}$ is the reduced temperature. Keeping only the

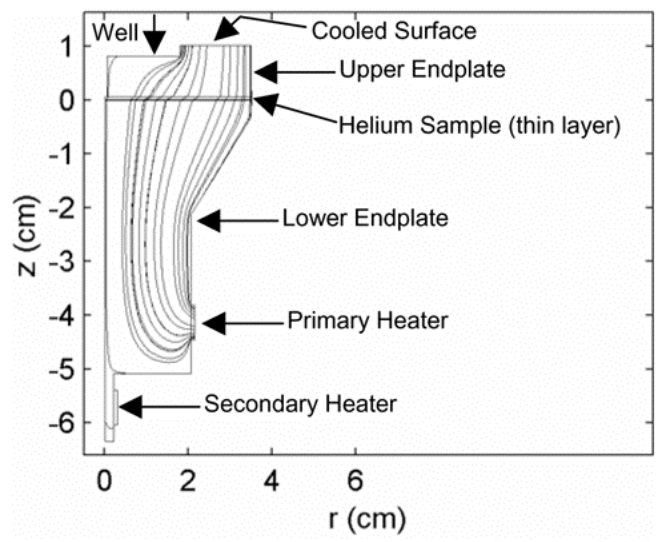

FIGURE 1. Cell model geometry with heat flow streamlines. The model is axisymmetric about $r=0$. Streamlines show heat flowing from the primary heater to the cooled surface. The principal cause of inhomogeneity of the heat flow in the helium is the "well" cut into the upper endplate to accommodate the diaphragm valve. 
lowest-order $\left(Q / Q_{c}\right)^{2}$ term of the expansion for $\Delta C_{Q}[2]$, and neglecting the variation of reduced temperature $t$ over the height of the cell, the fractional enhancement of $\Delta C_{Q}$ by inhomogeneity is

$$
E=\frac{\int t^{-\alpha}\left(Q(\mathbf{r}) / Q_{c}\right)^{2} d \mathbf{r}}{\int t^{-\alpha}\left(Q_{\text {nom }} / Q_{c}\right)^{2} d \mathbf{r}}=\frac{\int Q^{2}(\mathbf{r}) d \mathbf{r}}{Q_{\text {nom }}^{2} V_{\text {helium }}}
$$

where the integrals are taken over the helium volume, and $Q_{\text {nom }}$ is the "nominal" heat flux density (corresponding to that reported by Harter et al.) that would have been obtained for homogeneous heat flow.

In solutions of the linear heat flow equation for a given mixed boundary condition, the distribution of heat flux $\boldsymbol{Q}(\boldsymbol{r})$ is unaffected if all conductivities are scaled by the same factor. We have deliberately set $\kappa_{\mathrm{He}}$ so high that it is effectively infinite, thus $\boldsymbol{Q}(\boldsymbol{r})$ can depend only on the ratio of $R_{\mathrm{K}}$ to the endplate thermal conductivity $\kappa_{\mathrm{Cu}}$. The calculations confirm this scaling: values of $E$ agree to within $\sim 0.1 \%$ or better for scenarios where the product $R_{\mathrm{K}} \kappa_{C u}$ is equal.

Although it was impossible to deduce an accurate $R_{\mathrm{K}}$ from the Harter et al. data, extensive measurements[6] exist of the value and reproducibility of $R_{\mathrm{K}}$ for $\mathrm{Cu}$ surfaces and helium-II near $T_{\lambda}$. Those measurements, together with others made by us at the University of New Mexico and Caltech, show that an estimate of $R_{\mathrm{K}}=1.0 \pm 0.2 \mathrm{~cm}^{2} \mathrm{~K} / \mathrm{W}$ should be very reliable. We determined $\kappa_{C u}$ from published fits of $\kappa_{C u}(\mathrm{RRR})[7]$ and measurements of the RRR of several "core samples" cut from the bottom endplate of the Harter et al. cell by electrical discharge machining. These samples yielded RRR $=240-260$, thus $\kappa_{C u}=6.9$ $7.4 \mathrm{~W} / \mathrm{cmK}$.

The calculated $E$ is shown in Fig. 2. Using $R_{\mathrm{K}}=1 \mathrm{~cm}^{2} \mathrm{~K} / \mathrm{W}$ and $\kappa_{C u}=7.2 \mathrm{~W} / \mathrm{cm} \cdot \mathrm{K}$ yields $E=3.0$, compared to the observed anomalous enhancement of 7-12. Given the complexity and approximations involved in this post-experiment analysis, this level of agreement seems quite good.

Also shown in Fig. 2 are calculated maximum values of $Q_{\text {nom }} / Q_{c}$. Harter et al. found that above a maximum $Q_{\text {nom }} / Q_{c} \sim 0.3$ (their " $\beta$ " point) additional thermal resistance appeared between the bottom and top endplates. They proposed that this happened when the coherence length grew to exceed the surface roughness of the bottom endplate, effectively decreasing the bottom endplate area and increasing the apparent Kapitza resistance. Our present analysis provides another candidate explanation: the $\beta$ point might be occurring when the maximum value of $|Q(\boldsymbol{r})|$

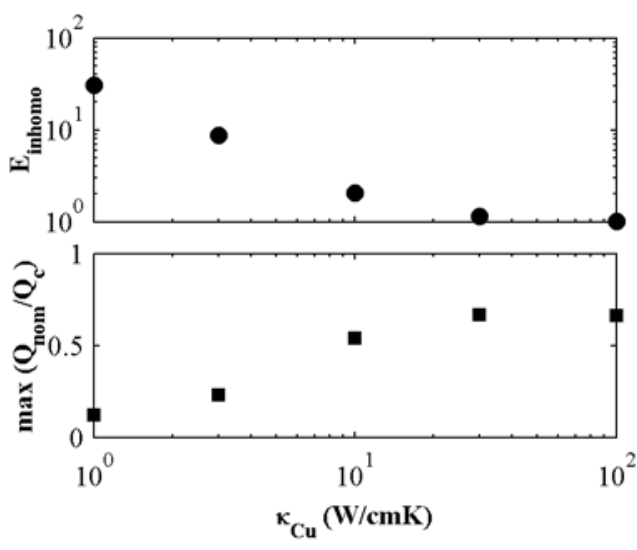

FIGURE 2. Calculated values of $E$ and the $\beta$ point over the expected range of $\kappa_{C u}$, assuming $R_{\mathrm{K}}=1 \mathrm{~cm}^{2} \mathrm{~K} / \mathrm{W}$.

becomes comparable to $Q_{c}$, causing a local breakdown of superflow. In this case $\max \left(Q_{\text {nom }} / Q_{c}\right)$ should equal the calculated $Q_{\text {nom }} / \max (|Q(\boldsymbol{r})|)$, which for our estimated values of $R_{\mathrm{K}}$ and $\kappa_{C u}$ is 0.43 , in reasonable agreement with observation. A recent reanalysis of some of the Harter et al. data by one of us (ARC) has shown that this new explanation may fit the data better than the correlation length argument.

In summary, numerical estimates of $\boldsymbol{Q}(\boldsymbol{r})$ in the Harter et al. cell indicate significant inhomogeneity which might explain both the anomalously large $\Delta C_{Q}$ and the $\beta$ point. There is a clear need for new measurements of $\Delta C_{Q}$ in a new cell with homogenous heat flow. Such a cell has been prepared at the University of New Mexico, and the cooldown is presently underway at Caltech. Work supported by NASA Fundamental Physics Discipline NAG3-1763 (STPB), NAG3-2900 (ARC, RAML, DLG), and JPL \#960494 (RVD). RVD acknowledges support at Caltech as a Moore Distinguished Scholar.

\section{REFERENCES}

1. Harter, A. W., Lee, R. A. M., Chatto, A., Wu, X., Chui, T. C. P., and Goodstein, D. L., Phys. Rev. Lett. 84, 2195 2198 (2000).

2. Chui, T. C. P. Goodstein, D. L., Harter, A. W., and Mukhopadhyay, R., Phys. Rev. Lett. 77, 1793-1796 (1996).

3. Haussmann, R., and Dohm, V., Czech. J. Phys. 46-S1, 171 (1996).

4. Boyd, S. T. P., and Goodstein, D. L., in preparation.

5. e.g. FEMLAB v2.3, COMSOL Inc, www.comsol.com.

6. Dingus, M., Zhong, F., and Meyer, H., J. Low Temp. Phys. 65, 185-212 (1986).

7. F. Pobell, Matter and Methods at Low Temperatures, Berlin: Springer-Verlag, 1992, p. 52. The WiedemannFranz inaccuracy $(\sim 10 \%$ here $)$ has negligible effect here. 